\title{
Actualización de la nomenclatura de los géneros Leptocoryphium Nees y Panicum L. (Gramineae-Paniceae) de la Flora del Paraguay
}

\author{
Fernando O. Zuloaga \& Osvaldo Morrone†
}

\section{Abstract}

ZULOAGA F. O. \& O. MORRONE (2014). An update of the nomenclature of the genera Leptocoryphium Nees and Panicum L. (Gramineae-Paniceae) of the Flora of Paraguay. Candollea 69: 89-91. In Spanish, English abstract.

The accepted names of Leptocoryphium Nees and Panicum L., published in 1994 in the fascicle Flora del Paraguay 23, are revised and updated following the most recent taxonomic revisions.

\section{Key-words}

GRAMINEAE - Leptocoryphium - Panicum - Floristic -

Paraguay 


\section{Introducción}

Recientes estudios filogenéticos basados en caracteres morfológicos y moleculares han cuestionado la monofilia de Panicum L. (Zuloaga \& al., 2000; Gómez-Martínez \& Culham, 2000; Giussani \& al., 2001; Duvall \& al., 2001; AliscionI $\&$ al., 2003). Estos estudios han reconocido géneros previamente incluidos bajo la sinonimia de Panicum como Dichanthelium (Hitchc. \& Chase) Gould (FreckMAnN \& Lelong, 2002), Steinchisma Raf. y Megathyrsus (Pilg.) B. K. Simon \& S. W. L. Jacobs (SIMON \& JACOBS, 2003) o varias especies fueron transferidas a géneros ya existentes, como por ejemplo Steinchisma o Hymenachne P. Beauv. (ZuloagA \& al., 1998; Aliscioni \& al., 2003) o a nuevos géneros de Paniceae (p. ej. Ocellochloa Zuloaga \& Morrone, Parodiophyllochloa Zuloaga \& Morrone [Morrone \& al., 2008; SedE \& al., 2009]) (ZULOAGA \& al., 2003).

\section{Nomenclatura actualizada}

Consecuentemente, junto con la publicación de la segunda y última parte de la tribu Paniceae (Fl. Paraguay 45, ZuloAGA \& al., 2014), actualizamos la nomenclatura publicada en la primera parte de la tribu (Fl. Paraguay 23, ZulOAGA \& al., 1994):

(Indicamos en negrita: los nombres acceptados actualmente y por la tanto nuevos con relación a Fl. Paraguay 23; en negrita y bastardilla, los nombres aceptados en $F l$. Paraguay 23, para los cuales hemos agregado el lugar de publicación en la Flora; en bastardilla, los basónimos cuando el nombre acceptado en $\mathrm{Fl}$. Paraguay 23 es una recombinación).

Anthaenantia P. Beauv., Ess. Agrostogr.: 48. 1812.

Anthaenantia lanata (Kunth) Benth. in J. Linn. Soc., Bot. 19: 39. 1881.

$\equiv$ Paspalum lanatum Kunth in Humb. \& al., Nov. Gen. Sp. 1, ed. folio: 78; ed. quarto: 94. 1816.

$\equiv$ Leptocoryphium lanatum (Kunth) Nees, Agrostol. Bras.: 84. 1829.

Véase Fl. Paraguay 23 (Zuloaga \& al., 1994: 194).

Dichanthelium (Hitchc. \& Chase) Gould in Brittonia 26: 59. 1974.

Dichanthelium sabulorum (Lam.) Gould \& C. A. Clark in Ann. Missouri Bot. Gard. 65: 1112. 1979.

$\equiv$ Panicum sabulorum Lam., Encycl. 4: 744. 1798.

Véase Fl. Paraguay 23 (Zuloaga \& al., 1994: 293).
Dichanthelium sabulorum var. polycladum (Ekman) Zuloaga in Amer. J. Bot. 90: 817. 2003.

$\equiv$ Panicum polycladum Ekman in Ark. Bot. 11(4): 24. 1912.

$\equiv$ Panicum sabulorum var. polycladum (Ekman) R. A. Palacios in Burkart, Fl. Il. Entre Ríos 2: 316. 1969.

Véase Fl. Paraguay 23 (Zuloaga \& al., 1994: 294).

Dichanthelium surrectum (Zuloaga \& Morrone) Zuloaga in Amer. J. Bot. 90: 817. 2003.

$\equiv$ Panicum surrectum Zuloaga \& Morrone in Novon 1: 111. 1991.

Véase Fl. Paraguay 23 (Zuloaga \& al., 1994: 308).

Hymenachne P. Beauv., Ess. Agrostogr.: 48. 1812.

Hymenachne grumosa (Nees) Zuloaga in Amer. J. Bot. 90: 817. 2003.

= Panicum grumosum Nees, Agrostol. Bras.: 182. 1829.

Véase Fl. Paraguay 23 (Zuloaga \& al., 1994: 236).

Hymenachne pernambucensis (Spreng.) Zuloaga in Amer. J. Bot. 90: 817. 2003.

$\equiv$ Agrostis pernambucensis Spreng., Syst. Veg. 1: 258. 1824.

$\equiv$ Panicum pernambucense (Spreng.) Pilg. in Engl. \& Prantl, Nat. Pflanzenfam. ed. 2, 14e: 15. 1940.

Véase Fl. Paraguay 23 (Zuloaga \& al., 1994: 278).

Megathyrsus (Pilg.) B. K. Simon \& S. W. L. Jacobs in Austrobaileya 6: 572. 2003.

Megathyrsus maximus (Jacq.) B. K. Simon \& S. W. L. Jacobs in Austrobaileya 6: 572. 2003.

= Panicum maximum Jacq., Collectanea 1: 76. 1787. Véase Fl. Paraguay 23 (Zuloaga \& al., 1994: 252).

Ocellochloa Zuloaga \& Morrone in Syst. Bot. 34: 688. 2009.

Ocellochloa stolonifera (Poir.) Zuloaga \& Morrone in Syst. Bot. 34: 690. 2009.

$\equiv$ Panicum stoloniferum Poir. in Lam., Encycl. Suppl. 4: 274. 1816.

Véase Fl. Paraguay 23 (Zuloaga \& al., 1994: 305). 
Parodiophyllochloa Zuloaga \& Morrone in Syst. Bot. 33: 69. 2008.

Parodiophyllochloa missiona (Ekman) Zuloaga \& Morrone in Syst. Bot. 33: 70. 2008.

= Panicum missionum Ekman in Ark. Bot. 11(4): 19. 1912.

Véase Fl. Paraguay 23 (Zuloaga \& al., 1994: 259).

Parodiophyllochloa ovulifera (Trin.) Zuloaga \& Morrone in Syst. Bot. 33: 73. 2008.

= Panicum ovuliferum Trin., Gram. Panic.: 191. 1826. Véase Fl. Paraguay 23 (Zuloaga \& al., 1994: 265).

Parodiophyllochloa pantricha (Hack.) Zuloaga \& Morrone in Syst. Bot. 33: 73. 2008.

$\equiv$ Panicum pantrichum Hack. in Verh. K. K. Zool.Bot. Ges. Wien 65: 72. 1915.

Véase Fl. Paraguay 23 (Zuloaga \& al., 1994: 268).

Steinchisma Raf. in Bull. Bot. (Geneve) 1:220. 1830.

Steinchisma decipiens (Trin.) W. V. Br. in Mem. Torrey Bot. Club 23(3): 20. 1977.

$\equiv$ Panicum decipiens Trin., Gram. Panic.: 227. 1826.

Véase Fl. Paraguay 23 (Zuloaga \& al., 1994: 225).

Steinchisma hians (Elliott) Nash in Small, Fl. S.E. U.S.: 105. 1903.

$\equiv$ Panicum hians Elliott, Sketch Bot. S. Carolina 1: 118. 1816.

Véase Fl. Paraguay 23 (Zuloaga \& al., 1994: 238).

Steinchisma laxa (Sw.) Zuloaga in Amer. J. Bot. 90: 817. 2003.

= Panicum laxum Sw., Prodr:: 23. 1788.

Véase Fl. Paraguay 23 (Zuloaga \& al., 1994: 246).

Steinchisma spathellosa (Döll) Renvoize in Kew Bull. 42: 921. 1987.

$\equiv$ Panicum spathellosum Döll in Mart., Fl. Bras. 2(2): 241. 1877.

Véase Fl. Paraguay 23 (Zuloaga \& al., 1994: 302).

\section{Referencias}

Aliscioni, S. S., L. M. Giussani, F. O. Zuloaga \& E. A. KellogG (2003). A molecular phylogeny of Panicum (Poaceae: Paniceae): tests of monophyly and phylogenetic placement within the Panicoideae. Amer. J. Bot. 90: 796-821.

Duvall, M. R., J. D. Noll \& A. H. Minn (2001). Phylogenetics of Paniceae (Poaceae). Amer. J. Bot. 88: 1988-1992.

Freckmann, R. W. \& M. G. Lelong (2002). Nomenclatural changes and innovations in Panicum and Dichanthelium (Poaceae: Paniceae). Sida 20: 161-174.

Giussani, L. M., J. H. Cota-Sánchez, F. O. Zuloaga \& E. A. KeLlOGG (2001). A molecular phylogeny of the grass subfamily Panicoideae (Poaceae) shows multiple origins of $\mathrm{C} 4$ photosynthesis. Amer. J. Bot. 88: 1993-2012.

Gómez-Martínez, R. \& A. Culham (2000). Phylogeny of the subfamily Panicoideae with emphasis on the tribe Paniceae: evidence from the trnL-F cpDNA region. In: JACOBS, S. W. L. \& J. EvERETT (ed.), Grasses: systematics and evolution: 136-140. CSIRO Publishing, Collingwood.

Morrone, O., S. S. Denham, S. S. Aliscioni \& F. O. Zuloaga (2008). Parodiophyllochloa, a new genus segregated from Panicum (Paniceae, Poaceae) based on morphological and molecular data. Syst. Bot. 33: 66-76.

Sede, S. M., F. O. Zuloaga \& O. Morrone (2009). Phylogenetic studies in the Paniceae (Poaceae-Panicoideae): Ocellochloa, a new genus from the New World. Syst. Bot. 34: 684-692.

Simon, B. K. \& S. W. L. JACOBS (2003). Megathyrsus, a new generic name for Panicum subgenus Megathyrsus. Austrobaileya 6: 571-574.

Zuloaga, F. O., O. Morrone, Z. E. Rúgolo de Agrasar, A. M. Anton, M. O. Arriaga \& A. M. Cialdella (1994). Gramineae V. Fl. Paraguay 23.

Zuloaga F. O., O. Morrone, A. S. Vega \& L. M. Giussani (1998). Revisión y análisis cladístico de Steinchisma (Poaceae: Panicoideae: Paniceae). Ann. Missouri Bot. Gard. 85: 631-656.

Zuloaga, F. O., O. Morrone \& L. M. Giussani (2000). A cladistic analysis of the Paniceae: a preliminary approach. In: JACOBS, S. W. L. \& J. Everett (ed.), Grasses: systematics and evolution: 123-135. CSIRO Publishing, Collingwood.

Zuloaga, F. O., O. Morrone, G. Davidse, T. S. Filgueiras, P. M. Peterson, R. J. Soreng \& E. J. Judziewicz (2003). Catalogue of New World Grasses (Poaceae): III. Subfamilies Panicoideae, Aristidoideae, Arundinoideae, and Danthonioideae. Contr. U. S. Natl. Herb. 46.

Zuloaga, F. O., O. Morrone \& J. F. Pensiero (2014). Gramineae VI. Fl. Paraguay 45. 